\title{
Temperature considerations on Hall Effect sensors current-related sensitivity behaviour
}

\author{
Maria-Alexandra Paun · Jean-Michel Sallese • \\ Maher Kayal
}

Received: 13 May 2013/Revised: 15 September 2013/Accepted: 23 September 2013/Published online: 15 October 2013

(C) Springer Science+Business Media New York 2013

\begin{abstract}
The present paper focuses on evaluating the temperature effects on Hall Effect sensors sensitivity behavior. To this purpose, an analysis of the factors affecting the sensors current-related sensitivity is performed, consisting of several pertinent considerations. An analytical investigation of the carrier concentration temperature dependence including the freeze-out effect influence was performed. This information was subsequently included in accurate prediction of the current-related sensitivity temperature behavior. For a specific CMOS integration process of the Hall sensors, a parabolic curve is obtained for the relative variation of the current-related sensitivity.
\end{abstract}

Keywords Hall Effect sensors - Temperature behavior · Freeze-out effect - Current-related sensitivity $\cdot$ Parabolic dependence

M.-A. Paun $(\varangle) \cdot$ J.-M. Sallese · M. Kayal

STI-IEL-Electronics Laboratory, Ecole Polytechnique Fédérale

de Lausanne (EPFL), Lausanne, Switzerland

e-mail: maria_paun2003@yahoo.com; map65@cam.ac.uk

J.-M. Sallese

e-mail: jean-michel.sallese@epfl.ch

M. Kayal

e-mail: maher.kayal@epfl.ch

Present Address:

M.-A. Paun

Department of Engineering, University of Cambridge,

Cambridge, UK

\section{Introduction}

The Hall Effect sensors are magnetic sensors which are used nowadays in a multitude of applications ranging from position detection, contactless switching, in DC brushless motors and as current-sensors in low-power applications [1]. Recent applications recommend these sensors for the detection of the superparamagnetic beads [2], for the measurements of the Brownian relaxation response of magnetic nanobeads in suspension [3], as well as for thermonuclear reactor magnetic diagnostics systems [4].

The performance of Hall Effect sensors resides in the highest sensitivity and the lowest offset. In recent papers, the authors analyzed the correlation between the Hall sensors geometry and their performance [5-8] and presented shapes $[9,10]$ that allowed offsets less than $30 \mu \mathrm{T}$ and drift less than $0.3 \mu \mathrm{T} /{ }^{\circ} \mathrm{C}$. The achieved performance for specific sensors in terms of offset and its drift are a few times better than the state-of-the-art. Research was conducted by the authors to predict Hall Effect sensors behavior by three-dimensional physical simulations [11] and guide the designer in optimal Hall sensor shape selection process.

The current work is intended to analyze the temperature behavior of Hall Effect sensors current-related sensitivity, by taking into account the variation of the specific parameters. Section 2 is devoted to introducing general considerations about the Hall sensors and the design of a specific cell in particular which exhibited the best performance. Section 3 is reserved for a detailed analysis of the current-related temperature dependence while Sect. 4 aims to present an analytical discussion of carrier concentration temperature dependence including the freeze-out effect. Finally, Sect. 5 summarizes the results of simulations for temperature coefficients of the specific parameters and 
ultimately provides the shape of the absolute-sensitivity curve with the temperature.

\section{Hall Effect sensors considerations}

\subsection{Hall voltage definition}

When polarizing a semiconducting device with a current and subjecting the probe to a magnetic field, the carriers are deviated under the influence of Lorentz force and a voltage difference appears between the opposite contacts in the form of the Hall voltage. The Hall voltage is given by the following relation, for a current polarization with $I_{\text {bias }}$ :

$V_{H}=G \frac{r_{H}}{n q t} I_{\text {bias }} B$

where $G$ is the geometrical correction factor, $r_{H}$ is the Silicon scattering factor, $n$ is the carrier density, $q$ is the elementary charge, $t$ is the thickness of the implantation profile and $B$ is the perpendicular component of the magnetic field induction, respectively [12].

From the equation above, we can see the need to use lightly doped semiconductors in order to achieve higher Hall voltages.

\subsection{Hall Effect sensors implementation}

In recent papers, the authors presented and analyzed nine integrated Hall sensors shapes in CMOS $0.35 \mu \mathrm{m}$ technology. Details about all the cells integration and performance tests can be found in [8-11]. In Fig. 1, precise design details

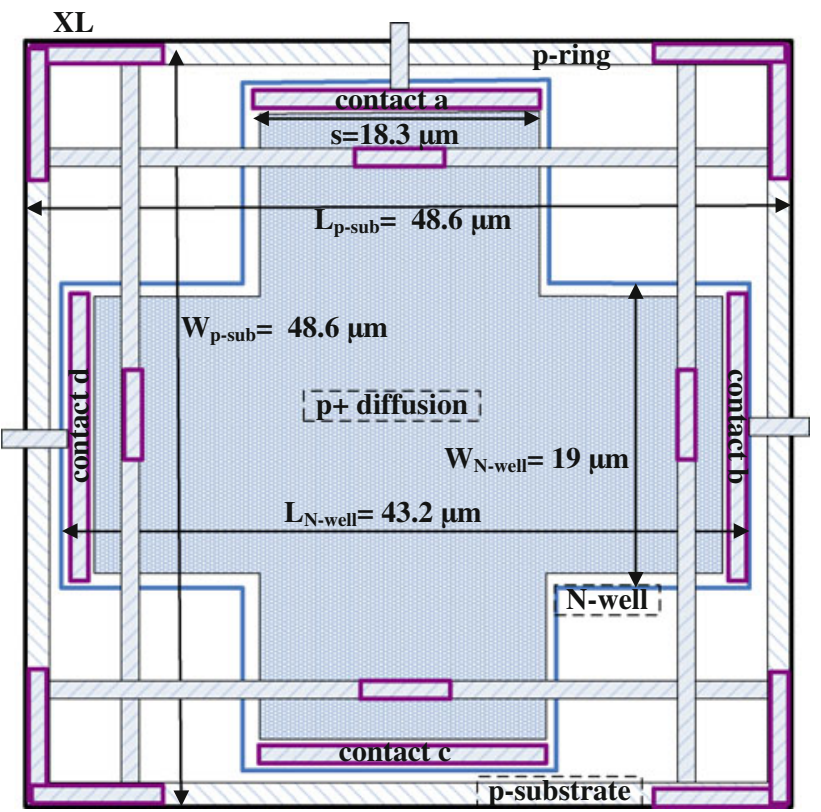

Fig. 1 The XL Hall cell detailed design parameters of the XL cell which displayed the maximum performance in terms of offset and its drift [9] are depicted. $W$ and $L$ stand for the width and length of the Hall sensor respectively, while $s$ denotes the contacts length. It is an $\mathrm{n}$-doped semiconductor structure, with $\mathrm{N}$-well implantation on a p-substrate. On top, to reduce the noise and to prevent the current to flow under the contacts, a p+ diffusion layer is also used.

\subsection{Hall cells absolute and current-related sensitivity}

The performance of the Hall cells is given by the analysis of their absolute sensitivity, the zero-magnetic field offset voltage and their corresponding temperature drifts [13, 14]. Therefore, in achieving Hall cells with good performance, a high sensitivity and a low offset drift are required.

An important figure of merit in Hall Effect sensors performance assessment is their sensitivity. The absolute sensitivity $S_{A}$ is defined as the ratio of the detected Hall voltage $V_{H}$ to the magnetic field induction, $B$. Moreover, one could also define the current-related sensitivity of the Hall sensor by the following relationship:

$S_{I}=\frac{S_{A}}{I_{\text {bias }}}=\frac{V_{H}}{B I_{\text {bias }}}=G \frac{r_{H}}{n q t_{\text {eff }}}$

When the doping density in the plate depends on the depth $z$, as in the case of ion-implanted layers, in Eqs. (1) and (2), the charge carrier density is a function of $z$, respectively $n=n(z)$.

The moment $m_{i}$ for an arbitrary index $i$ is computed as follows:

$m_{i}=\frac{\int_{0}^{\infty}\left(z-R_{p}\right)^{i} n(z) d z}{\int_{0}^{\infty} n(z) d z}$

The first order moment $R_{p}$ is:

$R_{p}=\frac{\int_{0}^{\infty} z n(z) d z}{\int_{0}^{\infty} n(z) d z}$

The standard deviation $\sigma$, the skewness $\gamma$ and the kurtosis $\beta$ are defined as:

$\sigma=\sqrt{m_{2}}, \gamma=\frac{m_{3}}{\sigma^{3}}, \beta=\frac{m_{4}}{\sigma^{4}}$

The ion implantation profile, using the moment parameters is expressed by:

$n(z)=n_{0} \exp \left[-\frac{\left(z-R_{p}\right)^{2}}{2 \sigma^{2}}\right]$

where $n_{0}$ is the peak concentration, which is defined with respect to the implantation dose $\Phi$ as:

$n_{0}=\frac{\Phi}{\sqrt{2 \pi} \sigma}$ 
A pure Gaussian distribution is obtained for $\gamma=0$ and $\beta=3$. In the case of $\gamma=0$ and $\beta=2$, the carrier concentration presents a strong flat region as in [15], respectively a quasi-constant concentration. Globally, these differences (for various values of $\beta$, also known as the effect of $\beta$ on profile shape) do not practically influence the carrier concentration. For $\gamma=0$, the distribution is symmetrical with respect to the axis $z=R_{p}$. In the simulation programs, a constant concentration can be however assumed tacitly, without producing major/ significant modifications in the results obtained.

Nevertheless, a Gaussian implantation profile was considered by the authors in a recent paper dealing with threedimensional simulation of the Hall cells [5], as well as the PhD thesis of Paun [7].

The sensitivity measurements were performed by subjecting the samples to a perpendicular magnetic field of $\mathrm{B}=0.497 \mathrm{~T}$. The absolute sensitivity for the XL Hall cell of Run 1 as a function of the biasing current, between 0 and $0.7 \mathrm{~mA}$, is presented in Fig. 2.

As presented in [3], the XL cell has $S_{I}=80.7 \mathrm{~V} / \mathrm{AT}$, where $G$ was computed for different structures by the authors [8]. This type of measurements was achieved with the automated setup and the data extracted is before the saturation occurs.

\subsection{Input resistance temperature dependence}

Measurements for the input resistance dependence with temperature of the XL integrated Hall cell is depicted in Fig. 3. The corresponding cell was placed in an oven and the temperature was cycled from -40 to $125^{\circ} \mathrm{C}$. The curve is presented with a second order fit.

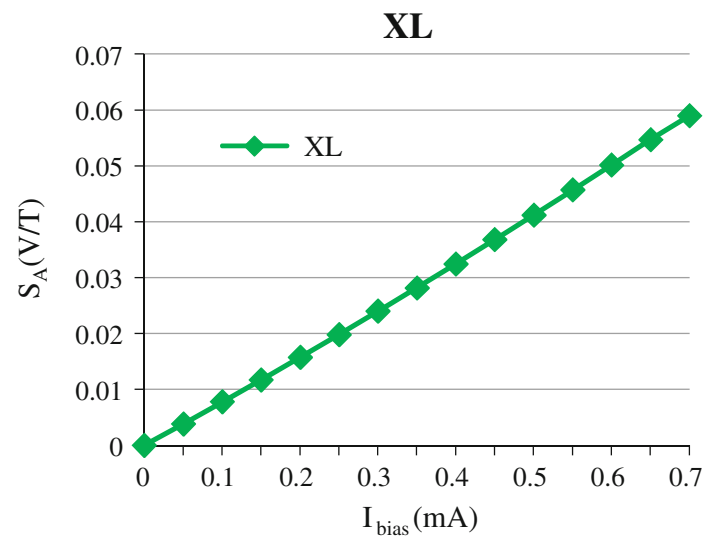

Fig. 2 The absolute sensitivity (V/T) of the XL cell versus the $I_{\text {bias }}$ $(\mathrm{mA})$

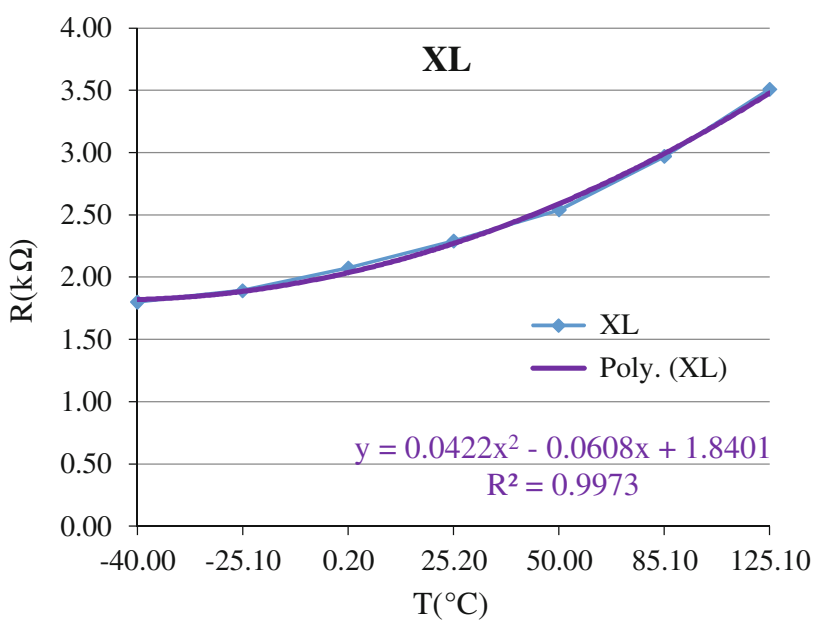

Fig. 3 Input resistance $R$ versus temperature for XL Hall cell

\section{Temperature dependence of the Hall cells current- related sensitivity}

From Eq. (2) above we can clearly see the factors that affect the absolute sensitivity temperature dependence:

$S_{I}(T)=G \frac{r_{H}(T)}{n(T) q t_{e f f}(T)}$

The current-related sensitivity increases with the current, as the effective thickness of the active area if the Hall plate is reduced.

Let us consider that $r_{H}=r_{H}(T), n=n(T)$ and $t_{\text {eff }}=$ $t_{\text {eff }}(T)$ are temperature dependent functions. We will have $S_{I}=S_{I}\left(r_{H}(T), n(T), t_{e f f}(T)\right)$, which means that it is an implicit function of $\mathrm{T}$.

Therefore, we can write:

$\frac{d S_{I}}{d T}=\frac{\partial S_{I}}{\partial r_{H}} \frac{\partial r_{H}}{\partial T}+\frac{\partial S_{I}}{\partial n} \frac{\partial n}{\partial T}+\frac{\partial S_{I}}{\partial t_{e f f}} \frac{\partial t_{e f f}}{\partial T}$

where $r_{H}, n$ and $t_{\text {eff }}$ are functions of a single variable "T", which makes the derivation operator $\frac{\partial}{\partial T}$ to become actually $\frac{d}{d t}$.

From Eq. (5), we obtain:

$$
\begin{aligned}
\frac{\partial S_{I}}{\partial r_{H}} & =\frac{G}{n q t_{e f f}} ; \frac{\partial S_{I}}{\partial n}=-\frac{G r_{H}}{n^{2} q t_{e f f}}=-S_{I} \frac{1}{n} ; \frac{\partial S_{I}}{\partial t_{e f f}}=-\frac{G r_{H}}{n q t_{e f f}^{2}} \\
& =-S_{I} \frac{1}{t_{e f f}}
\end{aligned}
$$

which replaced in Eq. (6) gives us:

$\frac{d S_{I}}{d T}=S_{I}\left(\frac{1}{r_{H}} \frac{d r_{H}}{d T}\right)-S_{I}\left(\frac{1}{n} \frac{d n}{d T}\right)-S_{I}\left(\frac{1}{t_{e f f}} \frac{d t_{e f f}}{d T}\right)$ 
We now make the specification that, by definition, the temperature coefficient of the physical quantity A, denoted by $\alpha_{A}$ is:

$\alpha_{A}=\frac{1}{A} \frac{d A}{d T}$

where $A$ is an element of the discrete set $A \varepsilon \boldsymbol{A} \equiv$ $\left\{S_{I}, r_{H}, n, t_{e f f}, \ldots\right\}$.

As presented in [12], the temperature coefficient $\alpha$ of a specific quantity $Q$ is defined as follows:

$\alpha=\frac{1}{Q_{0}} \frac{d Q}{d T}$

where $Q_{0}=Q\left(T_{o}\right)$ is the value of the specific quantity at room temperature $T_{o}=300 \mathrm{~K}$.

By consequence, the temperature coefficient $\alpha_{S_{I}}$ of the current-related sensitivity is the following:

$\alpha_{S_{I}}=\alpha_{r_{H}}-\alpha_{n}-\alpha_{t_{e f f}}$

In general, the effective thickness $t_{\text {eff }}$ can be defined as follows:

$t_{e f f}=t_{N W}-t_{p+}-w_{N W, s u b}-w_{N W, p+}$

where $t_{N W}$ is the depth of the $\mathrm{N}$-well implantation region, $t_{p+}$ is the thickness of the top $\mathrm{p}+$ layer, $w_{N W, \text { sub }}$ is the bottom depletion region between $\mathrm{N}$-well and the p-type substrate, $w_{N W, p+}$ is the upper depletion region between $\mathrm{N}$-well and the top $\mathrm{p}+$ layer [16].

As presented in a few papers published in the literature [1618], the effective thickness $t_{\text {eff }}$ is considered constant with the temperature, so its corresponding temperature coefficient is null, $\alpha_{t_{\text {eff }}}=0$. This assumption is valid when $V_{\text {substrate }}$ is constant and $V_{\text {output }}$ is in common mode, resulting from the presence of the common voltage at the input. Indeed some specific biasing conditions were used for Hall cells, as presented in details by the authors in a recent paper [9] where the automated measurement setup is presented. The substrate is biased with a constant voltage (either 0 or $3.3 \mathrm{~V}$ ), while the output voltage is collected with a lock-in amplifier.

However, the type of experimental data regarding the input resistance temperature dependence, in Fig. 3 could be useful when evaluating the temperature dependence of the effective thickness of the implantation profile, if needed. Nevertheless, this is not the objective of the present study and can be dealt with in a future paper.

A Taylor expansion of the function $S_{I}=S_{I}(T)$, around the value $T_{0}$, has the form:

$$
\begin{aligned}
S_{I}(T)= & S_{I}\left(T_{0}\right)+\left.\frac{1}{1 !} \frac{\partial S_{I}(T)}{\partial T}\right|_{T_{0}}\left(T-T_{0}\right) \\
& +\left.\frac{1}{2 !} \frac{\partial^{2} S_{I}(T)}{\partial T^{2}}\right|_{T_{0}}\left(T-T_{0}\right)^{2}+\left.\sum_{n=3}^{\infty} \frac{1}{n !} \frac{\partial^{n} S_{I}(T)}{\partial T^{n}}\right|_{T_{0}}\left(T-T_{0}\right)^{n}
\end{aligned}
$$

In the case in which:

$\left.\sum_{n=3}^{\infty} \frac{1}{n !} \frac{\partial^{n} S_{I}(T)}{\partial T^{n}}\right|_{T_{0}}\left(T-T_{0}\right)^{n}=O\left[\left(\left(T-T_{0}\right)^{n \geq 3}\right)\right] \rightarrow 0$

we have after a simple computation, the simplified version of Eq. (16):

$S_{I}(T)=S_{I}\left(T_{0}\right)+\alpha_{I}\left(T-T_{0}\right)+\beta_{I}\left(T-T_{0}\right)^{2}$

where

$\alpha_{I}=\left.\frac{1}{S_{I}\left(T_{0}\right)} \frac{\partial S_{I}(T)}{\partial T}\right|_{T_{0}}$

is the "first (order) coefficient" of the current-related sensitivity and

$\beta_{I}=\left.\frac{1}{2 S_{I}\left(T_{0}\right)} \frac{\partial^{2} S_{I}(T)}{\partial T^{2}}\right|_{T_{0}}$

is the "second (order) coefficient" of the current-related sensitivity, in the sense of a natural generalization of coefficients.

In the linear crop case, Eq. (18) becomes the following:

$S_{I}(T)=S_{I}\left(T_{0}\right)+\alpha_{I}\left(T-T_{0}\right)$

The temperature dependence of the magnetic sensitivity of the Hall cells is influenced by the temperature influence on two competing factors namely the freeze-out effect of the electron density and the Hall scattering coefficient respectively, as it has been investigated in the previous sections.

\section{Analytical investigation of the carrier concentration temperature dependence}

The carrier concentration temperature dependence was investigated in details, through an attentive analytical analysis including the consideration of the installation of freeze-out regime. The effect of carrier freeze-out on Hall sensors sensitivity was analyzed in a simplified way in [17]. The present study is more detailed than the latter one, giving the full set of precise analytical equations to be integrated in MATLAB for the complex carrier concentration temperature dependence. In addition to this, the computations are performed for a different CMOS integration technology.

In order to predict Hall Effect sensors sensitivity temperature behavior, one would need to calculate the temperature coefficient of the corresponding parameters that affect the sensitivity. By consequence, Eq. (14) needs to be taken into account. 
The intrinsic carrier concentration, $n_{i}$ is given by the following relationship, which depends on the effective density of states in the valence band and conduction band respectively:

$n_{i}(T)=\operatorname{Sqrt}\left(N_{C}(T) N_{V}(T)\right) \exp \left(\frac{-E_{g}}{2 k T}\right)$

where $N_{c}$ is the effective density of states in the conduction band, $N_{V}$ is the effective density of states in the valence band, $E_{g}$ is the bandgap of the semiconductor, $k$ is the Boltzmann constant and $T$ is the temperature in $\mathrm{K}$. In our case of Silicon, $E_{g}=1.12 \mathrm{eV}$.

$N_{c}$ and $N_{V}$ are both of them temperature dependent and obey the following equations:

$N_{C, V}(T)=2\left(\frac{2 \pi m_{n, p} k T}{h^{2}}\right)^{\frac{3}{2}}$

where $m_{n}$ is the mass of the donors $\left(\mathrm{e}^{-}\right), m_{p}$ is the mass of the acceptors (holes) and $h$ is the Planck constant. We know that $m_{n}=1.08 m_{e}$ and $m_{p}=0.81 m_{e}$ where $m_{e}$ is the elementary mass.

We also introduce the notion of freeze-out carrier concentration, $n_{f r}$, which is computed after the following relationship including temperature dependence:

$$
\begin{aligned}
n_{f r}(T)= & -\frac{\frac{N_{C}}{2} \exp \left(\frac{-E_{d}}{k T}\right)+N_{A}}{2}+\operatorname{Sqrt}\left(\left(\frac{\frac{N_{C}}{2} \exp \left(\frac{-E_{d}}{k T}\right)+N_{A}}{2}\right)^{2}\right. \\
& \left.+\left(N_{D}-N_{A}\right) \frac{N_{C}}{2} \exp \left(\frac{-E_{g}}{2 k T}\right)\right)
\end{aligned}
$$

where $E_{d}$ is the donor energy of the donor impurity, $N_{D}$ is the donor density and $N_{A}$ is the acceptor density, for the impurities with which the semiconductor is doped. The ionization energy of donor impurities is $E_{d}=0.049 \mathrm{eV}$ for Arsenic and $E_{d}=0.045 \mathrm{eV}$ for Phosphorus respectively [19].

The equation above is valid for temperatures which satisfy the relation:

$T \leq \frac{E_{g}}{k \ln N_{C} N_{V}-2 k \ln \left|N_{D}-N_{A}\right|}$

We need to calculate the minimum between the net impurity density and the freeze-out carrier concentration which is above computed. Further on, the electron density $n$, which interests us, will be given by the maximum between the latter quantity and the intrinsic density as shown by the formula:

$n=\operatorname{Max}\left(n_{i}, \operatorname{Min}\left(N_{D}-N_{A}, n_{f r}\right)\right)$

The detailed analysis of the carrier concentration temperature behavior gives us an insightful approach which will be used in accurate sensitivity prediction. The goal is to see how this ultimately affects the sensitivity of the Hall cells.

\section{Results and discussion}

5.1 The current-related sensitivity temperature behaviour

The temperature dependence of the magnetic sensitivity of the Hall cells is influenced by the temperature influence on two competing factors namely the freeze-out effect of the electron density and the Hall scattering coefficient respectively.

The values of the parameters used to simulate the Hall cells within CMOS Process 1 are summed up in Table 1.

Besides our integration process (Process 1), another CMOS process (Process 2) was simulated for the same cell. The data for Process 2 was taken from [20] and it is presented in Table 2. This data was used for the simulations obtained in Fig. 12.

In Fig. 4, the carrier concentration (in this case n-type carriers) is represented against the reciprocal temperature, for $N_{D}=8.16 \mathrm{E}+16 \mathrm{~cm}^{-3}$. From this graph we can see the three regions, namely intrinsic (yellow sector), extrinsic (or saturation) in the green sector and ionization (or freeze-

Table 1 Process 1 parameters used in the Hall cells simulation

\begin{tabular}{lll}
\hline Parameter & Symbol & Numerical value \\
\hline XL total length & $L_{\text {sensor }}$ & $43.2 \times 10^{-6} \mathrm{~m}$ \\
XL total width & $W_{\text {sensor }}$ & $19 \times 10^{-6} \mathrm{~m}$ \\
Thickness & $t$ & $10^{-6} \mathrm{~m}$ \\
Donor concentration & $N_{d}$ & $8.16 \times 10^{22} \mathrm{~m}^{-3}$ \\
Acceptor concentration & $N_{a}$ & $10^{21} \mathrm{~m}^{-3}$ \\
Conductivity & $\sigma$ & $933 \mathrm{Sm}^{-1}$ \\
Mobility & $\mu$ & $0.0715 \mathrm{~m}^{2} \mathrm{~V}^{-1} \mathrm{~s}^{-1}$ \\
Magnetic field & $B$ & $0.5 \mathrm{~T}$ \\
\hline
\end{tabular}

Table 2 Process 2 parameters used in the Hall cells simulation

\begin{tabular}{lll}
\hline Parameter & Symbol & Numerical value \\
\hline XL total length & $L_{\text {sensor }}$ & $43.2 \times 10^{-6} \mathrm{~m}$ \\
XL total width & $W_{\text {sensor }}$ & $19 \times 10^{-6} \mathrm{~m}$ \\
Donor concentration & $N_{d}$ & $3.3 \times 10^{22} \mathrm{~m}^{-3}$ \\
Acceptor concentration & $N_{a}$ & $10^{21} \mathrm{~m}^{-3}$ \\
Conductivity & $\sigma$ & $382.8 \mathrm{Sm}^{-1}$ \\
Mobility & $\mu$ & $0.1 \mathrm{~m}^{2} \mathrm{~V}^{-1} \mathrm{~s}^{-1}$ \\
Magnetic field & $B$ & $0.5 \mathrm{~T}$ \\
\hline
\end{tabular}




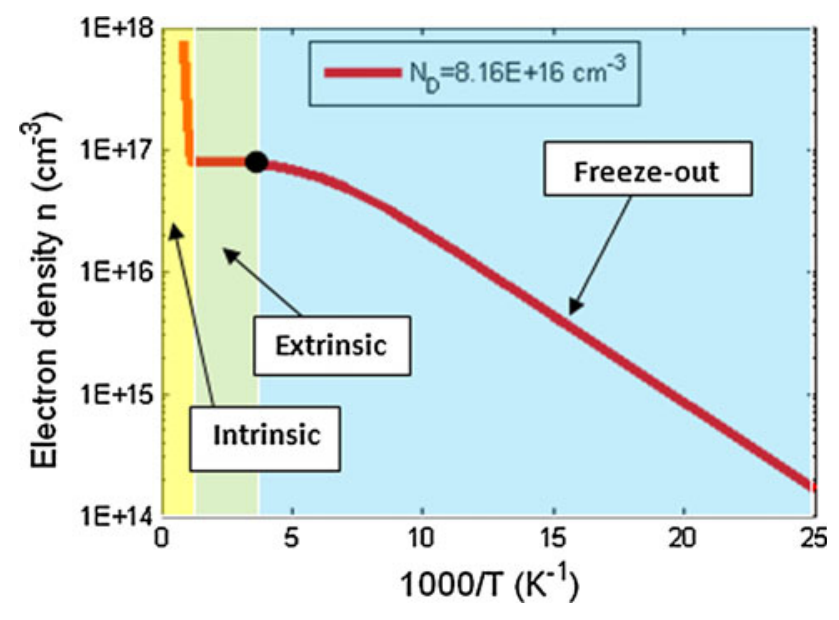

Fig. 4 Carrier density graphical representation as function of reciprocal temperature

out) in the blue sector. The point displayed on the curve corresponds to $n_{f r}$ which is obtained at the reciprocal of the freeze-out temperature, $T_{f r}$, more precisely $1,000 / T_{f r}$ abscissa point on the graph. In the case of our donor concentration, $T_{f r}=305 \mathrm{~K}$.

While the intrinsic region is identified in the zone where the carrier concentration increases with the temperature, the extrinsic region is where any increase in the temperature produces no increase in carrier concentration. To make it clear, the carrier density variation with temperature, due to reciprocal temperature, the graph in Fig. 4 should be "read" from right to left. In the very low temperatures range (equivalent to high $1 / \mathrm{T}$ ), negligible intrinsic electron-hole-pairs exist and the donor electrons are bound to the donor atoms, giving birth to the ionization region [19].

In the fabrication of Hall sensors, lower carrier concentrations yield higher sensitivity and in this case the freeze-out installs at lower temperatures. On the other hand, high carrier concentrations would have freeze-out temperatures approaching room temperature $T_{0}=300 \mathrm{~K}$.

The semi-log graph in Fig. 5 presents the electron density temperature dependence, plotted against the reciprocal temperature (equivalent to 1,000/T), for seven different levels of donor concentrations. The temperature $\mathrm{T}$ is into the interval from 35 to $1,200 \mathrm{~K}$ and the donor concentrations $N_{D}$ range from $10^{14}$ to $10^{17} \mathrm{~cm}^{-3}$. The graph is generated in MATLAB by using a code containing the full set of Eqs. (24-26).

For the specific CMOS process used in the integration of Hall cells, $\left(N_{D}=8.16 \mathrm{E}+16 \mathrm{~cm}^{-3}\right)$ the temperature coefficient $\alpha_{n}(\mathrm{ppm} / \mathrm{K})$ of the electron density $n$ has been calculated after extracting it from the curve in Fig. 4. The resulting $\alpha_{n}$ versus temperature is displayed in Fig. 6.

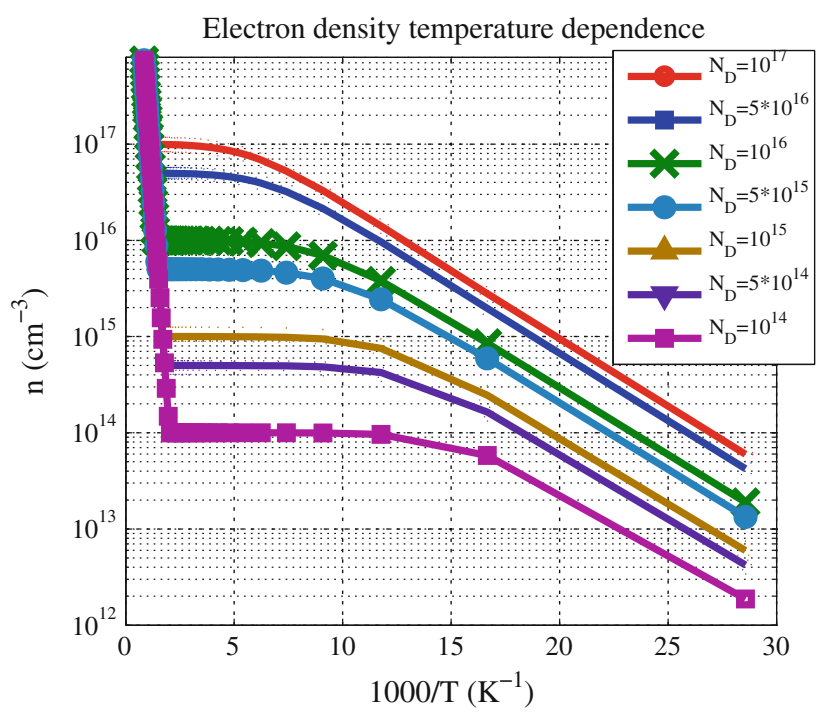

Fig. 5 Carrier concentration versus reciprocal temperature for different donor impurity concentrations

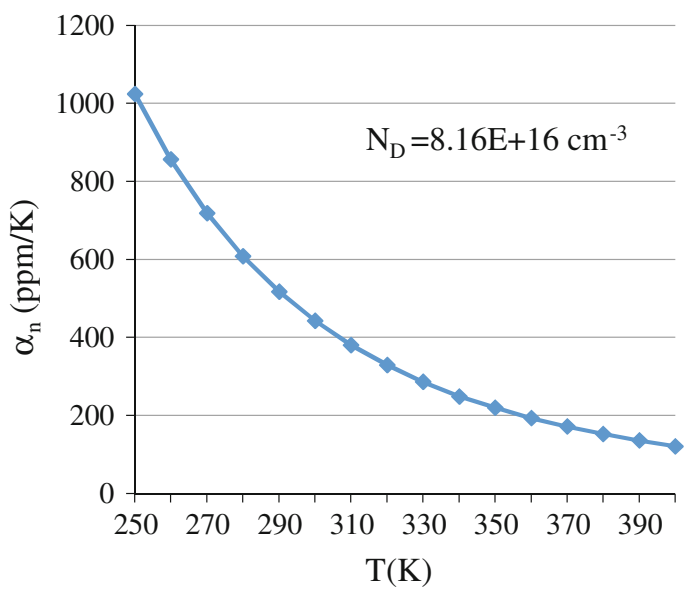

Fig. 6 Temperature coefficient $\alpha_{n}(\mathrm{ppm} / \mathrm{K})$ of $n$ versus temperature

In Fig. 6, we can observe that $\alpha_{n}$ decreases from $1,000 \mathrm{ppm} / \mathrm{K}$ for $250 \mathrm{~K}$ to around $120 \mathrm{ppm} / \mathrm{K}$ for the maximum temperature considered of $400 \mathrm{~K}$.

To obtain the temperature coefficient of the currentrelated sensitivity, we consider Eq. (8) with the addition that $t_{\text {eff }}$ is constant and we use the value of $r_{H}$ from [6]. Figure 7 depicts the obtained $\alpha_{S_{I}}$ plotted against the temperature. A zero temperature coefficient is obtained at $\mathrm{T}=312 \mathrm{~K}$, so around $\sim 40{ }^{\circ} \mathrm{C}$.

The relative variation $\gamma$ of the current-related sensitivity, $S_{I}$, with respect to the room temperature $T_{0}$ :

$\gamma=\frac{S_{I}(T)-S_{I}\left(T_{o}\right)}{S_{I}\left(T_{o}\right)}$ 


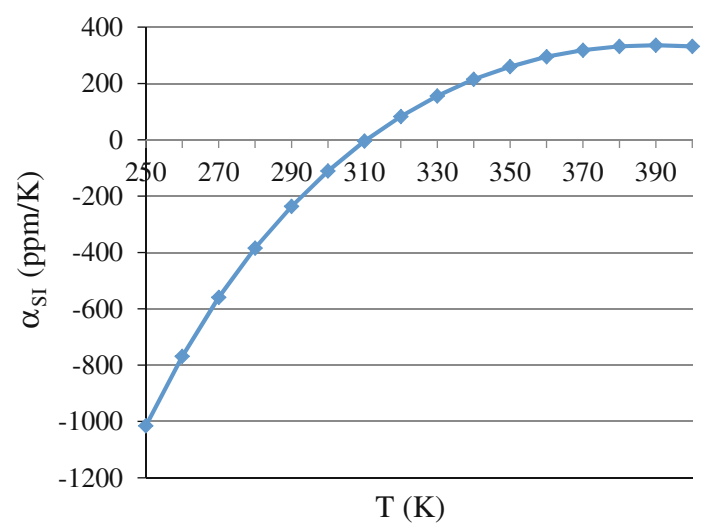

Fig. 7 Temperature coefficient $\alpha_{S_{I}}$ (ppm/K) of $S_{I}$ versus temperature

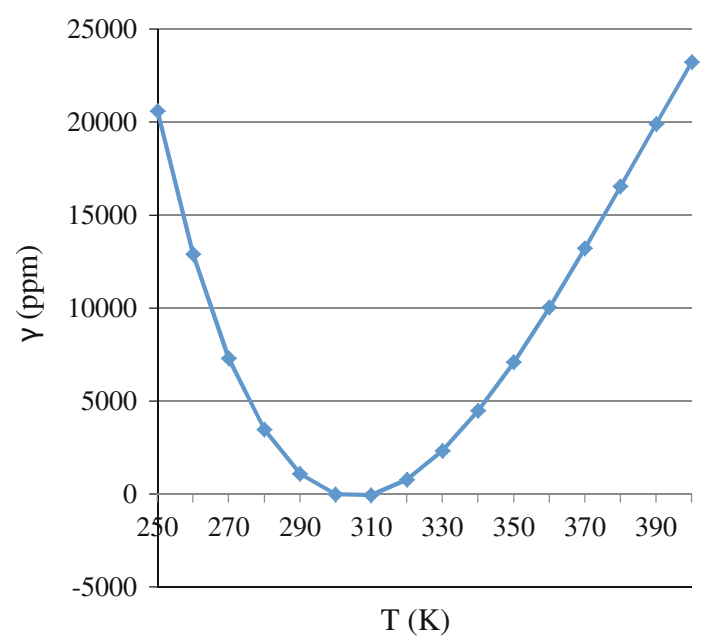

Fig. 8 The relative variation $\gamma(\mathrm{ppm})$ of the current-related sensitivity versus temperature

is displayed in Fig. 8 versus temperature.

We observe a parabolic dependence of the currentrelated sensitivity relative variation with a minimum around $305 \mathrm{~K}$. The drift of this relative variation is of maximum $24,000 \mathrm{ppm}$ over the whole considered temperature interval.

This parabolic trend result obtained is in agreement with the experimental results presented in the literature, in [17]. Similar measurements results for the current-related sensitivity temperature are obtained in [21], for Hall samples integrated in the same CMOS process as the ones discussed in the present study, for a temperature range of -40 to $80{ }^{\circ} \mathrm{C}$. In another recent paper by the authors [22], by using a circuit model developed for Hall cells performance assessment, a second order curve is also obtained for the current-related sensitivity temperature dependence and compared with the theoretical results.

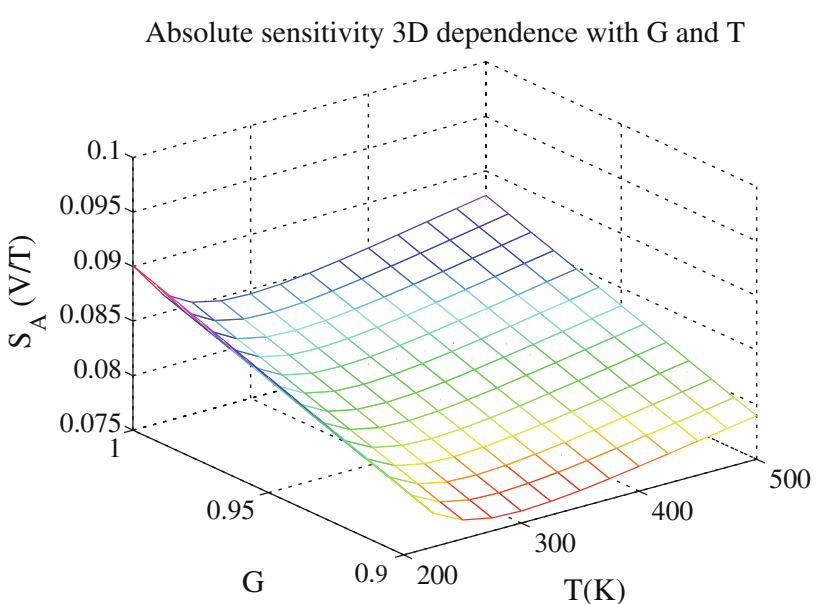

Fig. $9 \mathrm{~S}_{\mathrm{A}}=\mathrm{S}_{\mathrm{A}}(\mathrm{G}, \mathrm{T})$

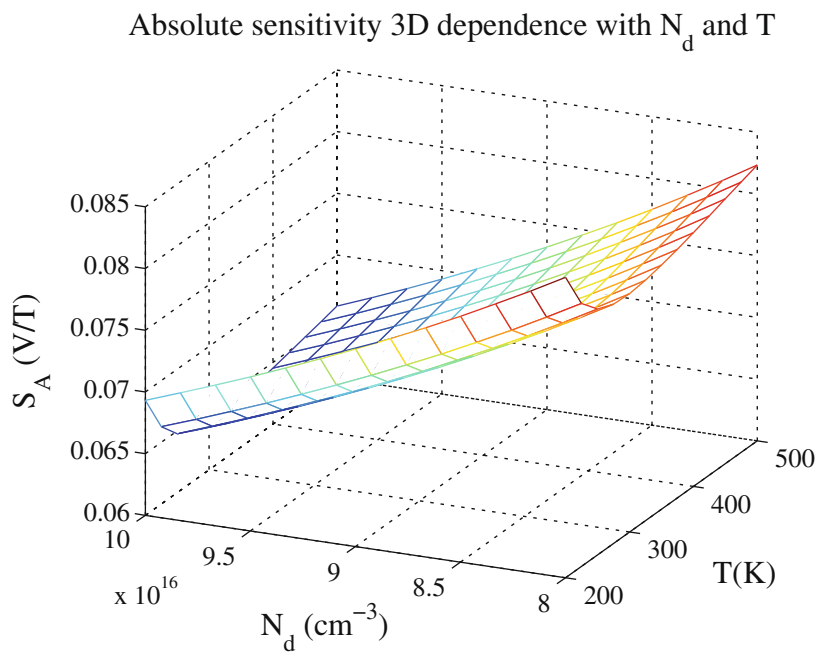

Fig. $10 \mathrm{~S}_{\mathrm{A}}=\mathrm{S}_{\mathrm{A}}\left(N_{d}, \mathrm{~T}\right)$

\subsection{Three-dimensional representation of the results}

Various three-dimensional representations of the simulations results were obtained in MATLAB. The focus is at this moment on the XL cell, but different integration processes are taken into account.

Figure 9 presents the three-dimensional representation of the absolute sensitivity as a function of both geometrical correction factor $G$ and absolute temperature, $\mathrm{S}_{\mathrm{A}}=\mathrm{S}_{\mathrm{A}}(\mathrm{G}, \mathrm{T})$.

Figure 10 depicts the three-dimensional representation of the absolute sensitivity as a function of both donor concentration $N_{d}$ and absolute temperature, $\mathrm{S}_{\mathrm{A}}=\mathrm{S}_{\mathrm{A}}\left(N_{d}, \mathrm{~T}\right)$.

The representation of the absolute sensitivity as a function of both $\mathrm{n}$-well implantation thickness $t$ and absolute temperature, $\mathrm{S}_{\mathrm{A}}=\mathrm{S}_{\mathrm{A}}(t, \mathrm{~T})$ is incorporated in Fig. 11. The thickness $t$ is considered from 1 to $2 \mu \mathrm{m}$, while $\mathrm{T}$ is ramped from 200 to $500 \mathrm{~K}$. 
Absolute sensitivity 3D dependence with $\mathrm{t}$ and $\mathrm{T}$

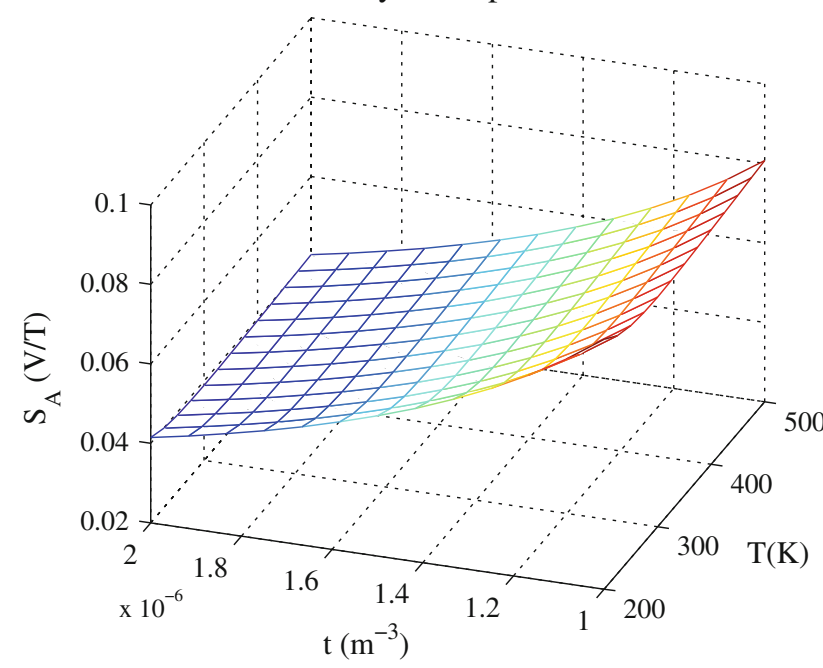

Fig. $11 \mathrm{~S}_{\mathrm{A}}=\mathrm{S}_{\mathrm{A}}(t, \mathrm{~T})$

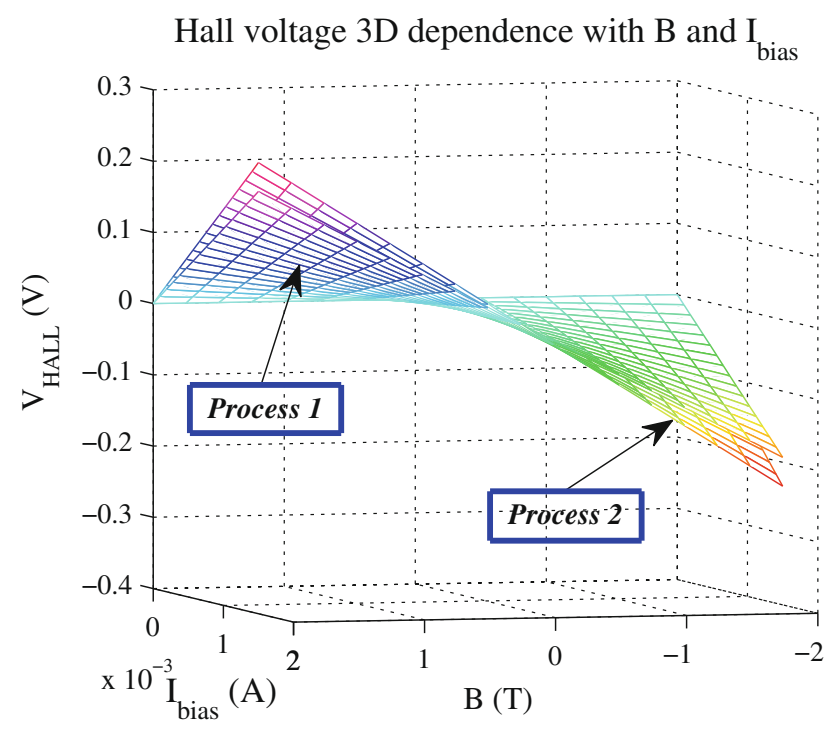

Fig. $12 \mathrm{~V}_{\mathrm{HALL}}=\mathrm{V}_{\mathrm{HALL}}\left(B, \mathrm{I}_{\text {bias }}\right)$

The Hall voltage as a function of magnetic field induction $B$ and biasing current is represented in Fig. 12, for two integration processes. The biasing current is ramped from 0 to $1 \mathrm{~mA}$ and the magnetic field $\mathrm{B}$ from -2 to $2 \mathrm{~T}$.

\section{Conclusions}

The work in the present paper was devoted to presenting a detailed analysis of the Hall cells current-related sensitivity temperature dependence. A certain Hall cell which provides high performance is presented in details. To evaluate its performance, experimental results for the sensitivity and input resistance temperature dependence were presented.

As one of the most important figure of merits in Hall cells performance is the current-related sensitivity and its temperature drift, an analytical procedure to assess this important parameter was proposed.

The current-related sensitivity temperature behavior of a particular Hall Effect sensor named XL is analyzed. To achieve this goal, all the parameters dependent with temperature were investigated. Analytical consideration of carrier concentration-temperature correlation reporting freeze-out effect was included. The drift of relative variation of the current-related sensitivity $\gamma$ is of maximum $24,000 \mathrm{ppm}$ over the entire considered temperature interval. The temperature dependence of $\gamma$ is a parabolic-type curve with a minimum at $305 \mathrm{~K}$. The results obtained are in accordance with the literature.

Three-dimensional representations of the absolute sensitivity dependence with the geometrical correction factor $G$ and absolute temperature, as well as its variation with the n-well implantation thickness $t$ and absolute temperature $T$ are generated. The absolute sensitivity variation with respect to both donor concentration $N_{d}$ and absolute temperature $T$ is also depicted. The Hall voltage as a function of magnetic field induction and biasing current is represented for two different integration processes.

Acknowledgments The first author, Maria-Alexandra Paun, wishes to thank the Swiss National Science Foundation (SNSF) from Switzerland for the promotion and encouragement of the scientific research of young doctors, respectively by providing the funding for her postdoctoral fellowship at Cambridge University.

\section{References}

1. Ramsden, E. (2006). Hall-Effect sensors: Theory and applications (2nd ed.). Amsterdam: Elsevier.

2. Manzin, A., Nabaei, V., \& Kazakova, O. (2012). Modelling and optimization of submicron Hall sensors for the detection of superparamagnetic beads. Journal of Applied Physics, 111(7), 07E513-07E513-3.

3. Osterberg, F. W., Rizzi, G., Gomez, Zardan, de la Torre, T., Stromberg, M., Stromme, M., et al. (2013). Measurements of Brownian relaxation of magnetic nanobeads using planar Hall effect bridge sensors. Biosensors \& Bioelectronics, 40(1), 147-152.

4. Bolshakova, I., Vasilevskii, I., Viererbl, L., Duran, I., Kovalyova, N., Kovarik, K., et al. (2013). Prospects of using in-containing semiconductor materials in magnetic field sensors for thermonuclear reactor magnetic diagnostics. IEEE Transactions on Magnetics, 49(1), 50-53.

5. Paun, M. A., Sallese, J. M., \& Kayal, M. (2013). Comparative study on the performance of five different Hall Effect devices, Sensors, ISSN 1424-8220, 13(2), 2093-2112.

6. Paun, M. A., Sallese, J. M., \& Kayal, M. (2013). Hall effect sensors design, integration and behaviour analysis. Journal of Sensors and Actuator Networks, 2(1), 85-97. 
7. Paun, M. A. (2013). Hall cells offset analysis and modeling approaches, $\mathrm{PhD}$ thesis, EPFL, Switzerland.

8. Paun, M. A., Sallese, J. M., \& Kayal, M. (2010). Geometry influence on Hall effect devices performance. UPB Scientific Bulletin, 72(4), 257-271.

9. Paun, M. A., Sallese, J. M., \& Kayal, M. (2011). Geometrical parameters influence on the Hall effect sensors offset and drift. In Proceedings of the 7th Conference on Ph.D. Research in Microelectronics and Electronics (PRIME), (pp. 145-148). Italy: IEEE.

10. Paun, M. A., Sallese, J. M., \& Kayal, M. (2012). A specific parameters analysis of CMOS Hall effect sensors with various geometries. In Proceedings of the 19th International Conference on Mixed Design of Integrated Circuits and Systems (MIXDES), (pp. 335-339), Varsaw: IEEE.

11. Paun, M. A., Sallese, J. M., \& Kayal, M. (2011). Hall effect sensors performance investigation using three-dimensional simulations. In Proceedings of the 18th International Conference on Mixed Design of Integrated Circuits and Systems (MIXDES), (pp. 450-455), Gliwice: IEEE.

12. Popovic, R. S. (2004). Hall effect devices (2nd ed.). Bristol, UK: Institute of Physics Publishing.

13. Blanchard, H., Iseli, C. D., \& Popovic, R. S. (1997). Compensation of the temperature-dependent offset drift of a Hall sensor. Sensors and Actuators A-Physical, 60(1-3), 10-13.

14. Blanchard, H., \& Popovic, R. S. (1999). On chip compensation of the temperature dependent offset drift of Hall sensors. In Proceeedings of the 10th International Conference on Solid-State Sensors and Actuators (Transducers'99), (pp. 598-601), Sendai, Japan.

15. Suzuki, K. (2010). Analysis of ion implantated profiles for accurate process/device simulation: Ion implantation profile database based on tail function. Fujitsu Scientific \& Technical Journal, 46(3), 307-317.

16. Xu, Y., \& Pan, H.-B. (2011). An improved equivalent simulation model for CMOS integrated Hall plates. Sensors, 11, 6284-6296.

17. Manic, D., Petr, J., \& Popovic, R. S. (2000). Temperature crosssensitivity of Hall plate in submicron CMOS technology. Sensors and Actuators A, 85, 244-248.

18. Demierre, M. (2003). Improvements of CMOS Hall Microsystems and Applications for Absolute Angular Position Measurements, Ph.D. Thesis, Ecole Polytechnique Federale de Lausanne, Lausanne, Switzerland.

19. Sze, S. M., \& Ng, K. K. (2007). Physics of semiconductor devices (3rd ed.). Hoboken, NJ: Wiley.

20. Dimitropoulos, P. D., Drljaca, P. M., Popovic, R. S., \& Chatznikolaou, P. (2008). Horizontal Hall devices: A lumped circuit model for EDA simulators. Sensors and Actuators A, 145-146, 161-175.

21. Ajbl, A., Pastre, M., \& Kayal, M. (2013). A fully integrated Hall sensor microsystems for contactless current measurement. IEEE Sensors Journal, 13(6), 2271-2278.

22. Paun, M. A., Sallese, J. M., \& Kayal, M. (2013). A Circuit Model for CMOS Hall Cells Performance Evaluation including Temperature Effect, Advances in Condensed Matter Physics, 2013, Article ID 968647, 10p.

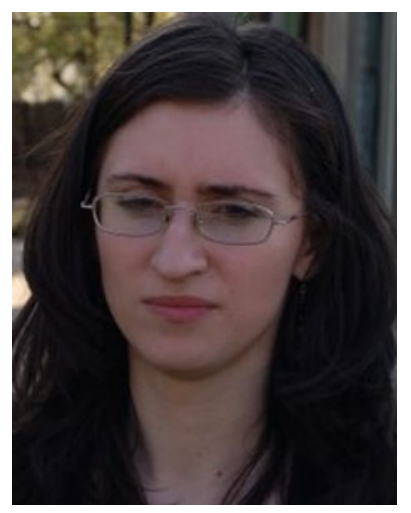

Maria-Alexandra Paun received the Engineer Diploma from Politehnica University of Bucharest, Faculty of Engineering in Foreign Languages, Electrical Engineering Division, English Stream, with a major in Computer Science, graduating top of her class. The Master Project was made in the Electronics Laboratory at Ecole Polytechnique Fédérale de Lausanne (EPFL, Switzerland) and treated a subject in the field of low dropout voltage regulators. Between October 2008 and April 2009, she was awarded a postgraduate Marie Curie research fellowship at the University of Kent at Canterbury, UK, where she worked in the field of Optical Coherence Tomography. Between May 2009 and March 2013, she was Assistant, Ph.D. student at EPF Lausanne and received her Ph.D. degree on the subject of Hall Effect sensors. She is currently a postdoctoral fellow at the Department of Engineering, at University of Cambridge, UK. Her main research interests are the analysis and modeling of sensors, development of circuit and three dimensional physical models. She is co-chair of IEEE Women in Engineering (WIE) Affinity Group in Switzerland and Secretary of IEEE Switzerland Section ExCom.

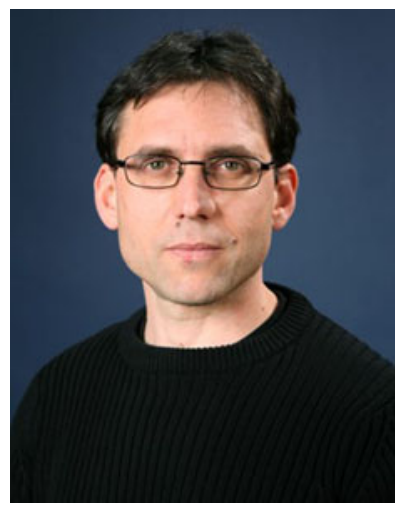

Jean-Michel Sallese received the M.Sc. degree from the Institut National des Sciences Appliquées (France) and the Ph.D. in physics from the University/ CNRS of Nice-Sophia Antipolis (France) where he worked on deep levels characterization in semiconductors. He joined the Swiss Federal Institute of Technology in Lausanne (EPFL) in 1991 where he worked on semiconductor laser diodes characterization and modeling. $\mathrm{He}$ is currently giving lectures on advanced semiconductor devices as and his research activities focus on compact modeling of multigate MOSFET's as well as modeling of Micro-Electro-Mechanical Systems. 


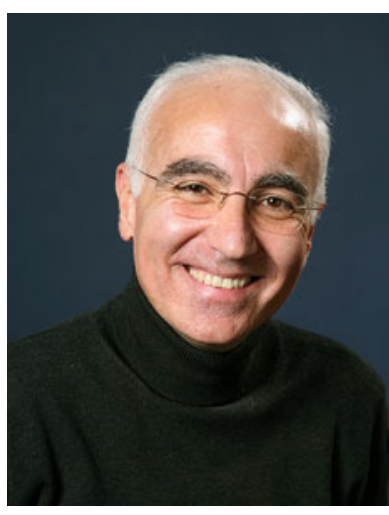

Maher Kayal received the M.S. and $\mathrm{Ph} . \mathrm{D}$. degrees in Electrical Engineering from the Ecole Polytechnique Fédérale de Lausanne (EPFL, Switzerland) in 1983 and 1989 respectively. He has been with the Electronics laboratories of the Ecole Polytechnique Fédérale de Lausanne (EPFL, Switzerland) since 1990, where he is currently a professor and vice dean for education of the school of engineering. He has published many scientific papers, is coauthor of three text books dedicated to mixed-mode CMOS design and he holds seven patents. His technical contributions have been in the area of analog and Mixed-signal circuits design including highly linear and tunable sensor microsystems, signal processing and CAD tools for analog design and layout automation. M. Kayal is a recipient of the Swiss Ascom award in 1990 for the best work in telecommunication fields. He is author and co-author of the following paper award in: ED\&TC conference in 1997, IEEE-AQTR in 2006, Mixdes conference in 2007 \& 2009, Powertech conference in 2009. M. Kayal received in 2009 the Swiss credit award for best teaching. 